\title{
Combination therapy for metastatic renal cell carcinoma
}

\author{
Carlo Buonerba $^{1}$, Giuseppe Di Lorenzo ${ }^{1}$, Guru Sonpavde $^{2}$ \\ ${ }^{1}$ University Federico II, Naples, Italy, ${ }^{2}$ Comprehensive Cancer Center, University of Alabama at Birmingham (UAB), Birmingham, AL, USA \\ Correspondence to: Guru Sonpavde, MD. Comprehensive Cancer Center, University of Alabama, Birmingham(UAB), 1720 2nd Ave. S., NP2540B, \\ Birmingham, AL 35294, USA. Email: gsonpavde@uabmc.edu.
}

\begin{abstract}
Current therapy for metastatic clear cell renal cell carcinoma (RCC) consists of the serial administration of single agents. Combinations of VEGF and mTOR inhibitors have been disappointing in previous randomized trials. However, the combination of lenvatinib, a multitargeted agent that inhibits VEGF as well as FGF receptors, and everolimus demonstrated promising results in a randomized phase II trial. Moreover, the emergence of programmed cell death 1 (PD-1) and programmed cell death ligand 1 (PD-L1) inhibitors has spawned the investigation of combinations of these agents with VEGF inhibitors and cytotoxic T-lymphocyte antigen 4 (CTLA-4) inhibitors. These ongoing phase III trials in conjunction with the development of predictive biomarkers and agents inhibiting novel therapeutic targets may provide much needed advances in this still largely incurable disease.
\end{abstract}

Keywords: Metastatic; renal cell carcinoma (RCC); combination therapy

Submitted Jan 21, 2016. Accepted for publication Jan 24, 2016.

doi: 10.21037/atm.2016.01.31

View this article at: http://dx.doi.org/10.21037/atm.2016.01.31

In 2016, renal cell carcinoma (RCC) will account for approximately 63,000 new cases and 14,000 deaths in the United States (1). Although virtually all patients with metastatic/inoperable kidney cancer eventually die of the disease, their median life expectancy has approximately doubled to 2 years with the advent of biological drugs targeting the VEGF (sunitinib, pazopanib, sorafenib, axitinib, bevacizumab) and the mTOR (everolimus, temsirolimus) pathways since 2005. Additional increments have occurred in 2015 in the salvage therapy space by the emergence of cabozantinib, a MET, AXL and VEGF receptor targeting tyrosine kinase inhibitor (TKI), and nivolumab, an anti PD-1 targeting monoclonal antibody, for patients with progression following VEGF inhibitors $(2,3)$. Cabozantinib extended median PFS (7.4 vs. 3.8 months, $\mathrm{P}<0.001)$ and nivolumab extended median OS $(25.0 \mathrm{vs}$. 19.6 months; $\mathrm{P}=0.002$ ), compared to everolimus.

The fibroblast growth factor (FGF) receptor appears to be a druggable biological target responsible for resistance to VEGF inhibitors in RCC $(4,5)$. Unfortunately, Dovitinib, a dual anti-VEGF and anti-FGF receptor TKI agent, did not prove effective in the third line setting after antiVEGF and anti-mTOR use compared to sorafenib, nor did it yield satisfactory results to warrant further investigation in combination with everolimus in the second line setting after VEGF inhibitors $(6,7)$. The combination of VEGF and mTOR inhibitors has yielded disappointing results as illustrated by randomized trials evaluating combinations of VEGF and mTOR inhibitors (everolimus or temsirolimus) (8-10). In addition, the combination of sunitinib and mTOR inhibitors (everolimus or temsirolimus) appeared prohibitively toxic in separate phase I trials $(11,12)$.

More recently, a more potent multitargeted TKI, lenvatinib, inhibiting pan-VEGF and FGF receptors has emerged (13). In contrast to the lack of feasibility of combinations of VEGF receptor TKIs and mTOR inhibitors, the combination of lenvatinib $18 \mathrm{mg}$ daily and everolimus $5 \mathrm{mg}$ daily was demonstrated to be feasible with manageable toxicities in a phase I trial (14). These results led to a randomized, open-label phase II trial by Motzer et al., in which 153 patients with advanced or metastatic, clear-cell RCC who had been treated with one line of VEGF inhibitor with progressive disease within 9 months of discontinuing the VEGF inhibitor were randomized in a 1:1:1 ratio to a continuous daily regimen of either lenvatinib ( $24 \mathrm{mg} /$ day), everolimus (10 mg/day) or lenvatinib plus 
everolimus (18 and $5 \mathrm{mg} /$ day, respectively) (15). The primary endpoint was PFS. Lenvatinib plus everolimus and lenvatinib alone were associated with a significantly longer median PFS of 14.6 and 7.4 months, respectively, compared to 5.5 months with everolimus. In an update, following recommendations of regulatory agencies, an independent radiological review (IRR) was reported (16). Median PFS by IRR was 12.8 months in the lenvatinib plus everolimus group, 9.0 months in the lenvatinib group, and 5.6 months in the everolimus group. PFS was significantly longer in patients that received lenvatinib plus everolimus compared to everolimus alone ( $\mathrm{HR}=0.45 ; \mathrm{P}=0.0029)$, but not for lenvatinib alone $v s$. everolimus $(\mathrm{HR}=0.62 ; \mathrm{P}=0.12)$. Notably, the smaller number of events assessed by IRR (62 events) than in the original report (75 events) appears to have played a major role in the lack of significance of the difference between lenvatinib and everolimus. IRR-assessed responses were achieved by $35 \%$ of patients who received lenvatinib plus everolimus, $39 \%$ of those who received lenvatinib alone and none of those who received everolimus alone. Median OS was 25.5 months for lenvatinib plus everolimus, 19.1 months for lenvatinib, and 15.4 months for everolimus in the updated analysis. The OS difference between lenvatinib plus everolimus $v s$. everolimus was significantly increased $(\mathrm{P}=0.024)$, but did not differ between lenvatinib and everolimus $(\mathrm{P}=0.12)$. Similar numbers of patients $(28 \%$ to $36 \%)$ in each arm received post-study treatment.

A total of $71 \%$ of patients assigned to lenvatinib plus everolimus, and $62 \%$ of those assigned to single-agent lenvatinib needed a lenvatinib dose reduction due to toxicities. In contrast, $2 \%$ of patients assigned lenvatinib plus everolimus and $26 \%$ of patients assigned everolimus alone needed an everolimus dose reduction. When examining discontinuations, $24 \%$ of patients assigned lenvatinib plus everolimus, $25 \%$ of patients allocated lenvatinib, and $12 \%$ of patients who received single-agent everolimus discontinued therapy due to adverse events (AEs). Grade 3 and 4 events occurred in $50 \%$ of patients receiving everolimus $(50 \%), 79 \%$ of those receiving lenvatinib and $71 \%$ of those receiving lenvatinib plus everolimus. The most common grade 3 therapy-related AEs included diarrhea, fatigue or asthenia, and hypertension in patients receiving lenvatinib plus everolimus; proteinuria, hypertension, and diarrhea in those receiving lenvatinib alone and anemia, dyspnea, hypertriglyceridaemia, and hyperglycemia in those receiving everolimus alone. Notably, hypothyroidism was also observed in lenvatinib containing arms. Fatal AEs occurred in one patient administered lenvatinib plus everolimus (cerebral haemorrhage), three patients assigned single-agent lenvatinib (myocardial infarction, judged possibly related to study treatment; and intracranial hemorrhage and sepsis, neither considered treatment-related) and two patients receiving everolimus alone (acute respiratory failure and sepsis, neither treatment-related).

These data require validation and encourage further investigation of the combination, but they have to be interpreted with caution in view of a number of limitations of the trial. First, this was a randomized phase II trial of modest size and was not double-blinded. However, the authors did update their results by an IRR assessment, which corroborated the increment provided by the combination of lenvatinib and everolimus. Second, although groups were balanced for major prognostic factors, the small sample size makes it impossible to control and account for imbalances of other potential confounders, especially molecular factors. Indeed, some clinical factors such as the proportion of patients who had $\geq 3$ metastases and who had received sunitinib were different between the groups. Third, quality of life and patient reported outcomes were not reported, which is essential to evaluate the risk/ benefit ratio of combined $v s$. single-agent treatment in the context of increased toxicities. Fourth, although the PFS associated with lenvatinib plus everolimus exceeded 1 year, the median duration of treatment was 7.6 months, which was similar to the median duration of lenvatinib alone (7.4 months) and only modestly longer than the median duration of everolimus alone (4.1 months). In this regard, a significant proportion of patients interrupted lenvatinib in the combination arm for reasons other than progressive disease, while everolimus appears to have been continued. Thus, the impressive PFS of the combination arm may be accounting for therapy using the combination followed by everolimus alone.

Optimally, the impressive results above should be corroborated by a prospective, randomized phase III trial in post VEGF inhibitor patients using lenvatinib plus everolimus in the investigational arm and either axitinib, cabozantinib or nivolumab in the comparator arm employing OS as the primary end point. Moreover, the emergence of T-cell checkpoint inhibitors has spawned other promising combinations. Indeed, combinations exploit the possibility of synergistic activity and overcome the difficulty of delivering multiple agents sequentially as monotherapy owing to decline in performance status and 
Table 1 Ongoing or planned randomized phase III trials of combination therapy for metastatic clear cell RCC

\begin{tabular}{lcccc}
\hline Trial ID & Phase & Line of therapy & Standard arm & Experimental arm \\
\hline NCT02231749 & III & First & Sunitinib & Nivolumab + ipilimumab \\
(CHECKMATE-214) & & & \\
NCT02420821 (IMmotion151) & III & First & Sunitinib & Bevacizumab + atezolizumab $^{\text {NCT01582672 }}$ \\
Pending & III & First & Sunitinib & Sunitinib + AGS-003 \\
Pending & III & First & Sunitinib & Axitinib + avelumab \\
\hline
\end{tabular}

*, AGS-003 is an immunotherapeutic that employs RNA isolated from the primary tumor to program autologous dendritic cells to induce tumor-specific immunity. RCC, renal cell carcinoma.

comorbidities. For example the combination of nivolumab and ipilimumab is being compared with sunitinib as firstline therapy in a phase III U.S Intergroup trial (Table 1). However, the combination of ipilimumab and nivolumab does have substantial toxicities and warrants careful patient selection (17). Combinations of VEGF receptor TKIs, sunitinib or pazopanib, and PD-1 pathway inhibitors have also preliminarily demonstrated increased gastrointestinal and hepatic toxicities, although the combination of axitinib and PD-1 inhibition may be feasible $(18,19)$. Indeed, a phase III trial is planned to compare the combination of axitinib plus avelumab (a PD-L1 inhibitor) vs. sunitinib as firstline therapy. Moreover, the combination of bevacizumab and PD-L1 inhibition (atezolizumab) appears feasible, which has led to an ongoing phase III trial comparing this combination $v s$. sunitinib as first-line therapy (20). Additionally, a different strategy of developing and studying optimal sequencing of single agents is essential, since a substantial proportion of patients in the community may be ineligible for these somewhat toxic combinations due to comorbidities or suboptimal performance status. Finally, as we develop novel agents and tolerable rational combinations, it is imperative to co-develop predictive biomarkers to enable precision medicine.

\section{Acknowledgements}

None.

\section{Footnote}

Provenance: This is a Guest Commentary commissioned by Guest Editor Xiongbing Zu, MD, PhD (Department of Urology, Xiangya Hospital, Central South University, Changsha, China).
Conflicts of Interest: C Buonerba: Research support from Sanofi. G Di Lorenzo: Research support from Sanofi. G Sonpavde: Research support to institution from Bayer, Boehringer-Ingelheim and Onyx; Consultant for Merck, Bayer, Sanofi, Genentech, Pfizer, Argos and Novartis.

\section{References}

1. Siegel RL, Miller KD, Jemal A. Cancer statistics, 2016. CA Cancer J Clin 2016;66:7-30.

2. Choueiri TK, Escudier B, Powles T, et al. Cabozantinib versus Everolimus in Advanced Renal-Cell Carcinoma. N Engl J Med 2015;373:1814-23.

3. Motzer RJ, Escudier B, McDermott DF, et al. Nivolumab versus Everolimus in Advanced Renal-Cell Carcinoma. N Engl J Med 2015;373:1803-13.

4. Rini BI. New strategies in kidney cancer: therapeutic advances through understanding the molecular basis of response and resistance. Clin Cancer Res 2010;16:1348-54.

5. Sonpavde G, Willey CD, Sudarshan S. Fibroblast growth factor receptors as therapeutic targets in clear-cell renal cell carcinoma. Expert Opin Investig Drugs 2014;23:305-15.

6. Motzer RJ, Porta C, Vogelzang NJ, et al. Dovitinib versus sorafenib for third-line targeted treatment of patients with metastatic renal cell carcinoma: an open-label, randomised phase 3 trial. Lancet Oncol 2014;15:286-96.

7. Powles T, Foreshew SJ, Shamash J, et al. A phase Ib study investigating the combination of everolimus and dovitinib in vascular endothelial growth factor refractory clear cell renal cancer. Eur J Cancer 2014;50:2057-64.

8. Rini BI, Bellmunt J, Clancy J, et al. Randomized phase III trial of temsirolimus and bevacizumab versus interferon alfa and bevacizumab in metastatic renal cell carcinoma: INTORACT trial. J Clin Oncol 2014;32:752-9. 
9. Ravaud A, Barrios CH, Alekseev B, et al. RECORD-2: phase II randomized study of everolimus and bevacizumab versus interferon $\alpha-2 \mathrm{a}$ and bevacizumab as first-line therapy in patients with metastatic renal cell carcinoma. Ann Oncol 2015;26:1378-84.

10. Flaherty KT, Manola JB, Pins M, et al. BEST: a randomized phase II study of vascular endothelial growth factor, RAF kinase, and mammalian target of rapamycin combination targeted therapy with bevacizumab, sorafenib, and temsirolimus in advanced renal cell carcinoma--a trial of the ECOG-ACRIN Cancer Research Group (E2804). J Clin Oncol 2015;33:2384-91.

11. Molina AM, Feldman DR, Voss MH, et al. Phase 1 trial of everolimus plus sunitinib in patients with metastatic renal cell carcinoma. Cancer 2012;118:1868-76.

12. Patel PH, Senico PL, Curiel RE, et al. Phase I study combining treatment with temsirolimus and sunitinib malate in patients with advanced renal cell carcinoma. Clin Genitourin Cancer 2009; 7:24-7.

13. Matsui J, Yamamoto Y, Funahashi Y, et al. E7080, a novel inhibitor that targets multiple kinases, has potent antitumor activities against stem cell factor producing human small cell lung cancer H146, based on angiogenesis inhibition. Int J Cancer 2008;122:664-71.

14. Molina AM, Hutson TE, Larkin J, et al. A phase $1 \mathrm{~b}$ clinical trial of the multi-targeted tyrosine kinase inhibitor lenvatinib (E7080) in combination with everolimus for treatment of metastatic renal cell carcinoma (RCC).

Cite this article as: Buonerba C, Di Lorenzo G, Sonpavde G. Combination therapy for metastatic renal cell carcinoma. Ann Transl Med 2016;4(5):100. doi: 10.21037/atm.2016.01.31
Cancer Chemother Pharmacol 2014;73:181-9.

15. Motzer RJ, Hutson TE, Glen H, et al. Lenvatinib, everolimus, and the combination in patients with metastatic renal cell carcinoma: a randomised, phase 2, open-label, multicentre trial. Lancet Oncol 2015;16:1473-82.

16. Motzer RJ, Hutson TE, Ren M, et al. Independent assessment of lenvatinib plus everolimus in patients with metastatic renal cell carcinoma. Lancet Oncol 2016;17:e4-5.

17. Larkin J, Chiarion-Sileni V, Gonzalez R, et al. Combined nivolumab and ipilimumab or monotherapy in untreated melanoma. N Engl J Med 2015;373:23-34.

18. Amin A, Plimack ER, Infante JR, et al. Nivolumab (anti-PD-1; BMS-936558, ONO-4538) in combination with sunitinib or pazopanib in patients (pts) with metastatic renal cell carcinoma (mRCC). J Clin Oncol 2014;32:abstr 5010.

19. Atkins MB, Gupta S, Choueiri TK, et al. Phase Ib dosefinding study of axitinib plus pembrolizumab in treatmentnaïve patients with advanced renal cell carcinoma. J Immunother Cancer 2015;3:353.

20. Sznol M, McDermott DF, Jones S, et al. Phase Ib evaluation of MPDL3280A (anti-PDL1) in combination with bevacizumab (bev) in patients (pts) with metastatic renal cell carcinoma (mRCC). J Clin Oncol $2015 ; 33$ :abstr 410 . 